\title{
Emerging Role of Hypofractionated Radiotherapy with Simultaneous Integrated Boost in Modern Radiotherapy of Breast Cancer
}

\author{
Mirko Nitsche $^{a, b} \quad$ Juergen Dunst ${ }^{b} \quad$ Ulrich M. Carl ${ }^{a} \quad$ Robert M. Hermann ${ }^{a, c}$ \\ a Zentrum für Strahlentherapie und Radioonkologie, Bremen/Westerstede, Germany; \\ ${ }^{b}$ Klinik für Strahlentherapie, Karl-Lennert-Krebscentrum, Universität Kiel, Germany; \\ ${ }^{c}$ Abteilung Strahlentherapie und Spezielle Onkologie, Medizinische Hochschule Hannover, Germany
}

Keywords

Breast cancer · Hypofractionation · Radiotherapy

\section{Summary}

Hypofractionated radiotherapy for breast cancer is becoming increasingly important. The scientific background of this development as well as the introduction of the simultaneous integrated boost to the primary tumor region in this context are discussed here.

(c) 2015 S. Karger GmbH, Freiburg

\section{Introduction}

Current standard whole breast irradiation (WBI) in breast-conserving concepts consists of a total dose (TD) of 50 Gy delivered in 25 daily fractions over a period of approximately 35 days. Alternatively, 50.4 Gy are applied using a daily dose of 1.8 Gy. An additional boost to the primary tumor region at a TD of $12-16 \mathrm{~Gy}$ is indicated in the majority of patients, resulting in an overall treatment period of up to 47 days [1-4]. Impressive data of more than 10,000 women in 17 randomized trials with a 15 -year follow-up confirm a risk reduction of ipsilateral breast recurrences by a factor of at least 3-4 [5]. This translates into a risk reduction of about $3 \%$ for breast cancer deaths in node-negative patients and about $8 \%$ in node-positive patients. Moreover, there is plenty of experience concerning treatment-related acute and late toxicity accompanied by excellent cosmetic results [1].

\section{KARGER}

Fax +497614520714

\section{(c) 2015 S. Karger GmbH, Freiburg}

$1661-3791 / 15 / 0105-0320 \$ 39.50 / 0$

\author{
Is There a Rationale for Changing the Established \\ Fractionation Regimens in the Near Future?
}

Malignant tumor cells and the surrounding healthy tissues differ with regard to their radiation sensitivity. The cells of normal healthy tissues are able to recover from sublethal radiation damage, whereas tumor cells show limited repair. These differences have been investigated intensively and have resulted in distinctive radiotherapeutic treatment regimens in which the daily fraction dose, $\mathrm{TD}$, and adherent treatment schedules have been established [6]. Dose effectiveness in modern radiotherapy is described with the linear-quadratic model ( $\alpha / \beta$ model), predicting radiobiologically equivalent (isoeffective) doses. The $\alpha / \beta$ value of the linear-quadratic model describes the sensitivity of healthy tissue and tumor cells depending on fractionation and TD. The fractionation sensitivity of any tissue is characterized by its $\alpha / \beta$ value; the $\alpha / \beta$ value is a mathematically calculated term derived from the dose-effect curve of the tissue, given in units of Gray (Gy). Generally, the $\alpha / \beta$ values of healthy tissues for late reactions are low (in the range of 2-3 Gy). In contrast, tumors and early reacting tissues have much higher $\alpha / \beta$ values in the range of $10 \mathrm{~Gy}$ or more [7]. In order to protect the healthy tissue from late radiation effects, irradiation should therefore be administered at low single doses of 1.2-2 Gy.

The linear-quadratic model has been confirmed in a variety of randomized studies, and it is a proven radiobiological fact that the greatest therapeutic window with regard to both local tumor control and late tissue effects is achieved if low doses per fraction are administered. However, the dogma that all tumors have high $\alpha / \beta$ values has been challenged over the past years. Over the past decade, growing evidence has accumulated that at least some cancers may have exceptionally low $\alpha / \beta$ values. This has so far been proven 
for prostate cancer with an $\alpha / \beta$ value of about $1.5 \mathrm{~Gy}$. If the $\alpha / \beta$ value of the tumor is below the $\alpha / \beta$ value of late-reacting surrounding tissue, treatment with higher doses per fraction is, from a theoretical point of view, advantageous. Therefore, hypofractionation has a well-defined theoretical basis in prostate cancer, and the results of completed and ongoing randomized studies support the notion that at least moderate hypofractionation is feasible in prostate cancer. Moreover, recent data suggest that prostate cancer is probably not the only exception and that some form of hypofractionation may be feasible in certain other cancer entities [8].

\section{Why Was Hypofractionation Introduced in Breast Cancer?}

The hypofractionation studies in breast cancer were started at a time when the data on low $\alpha / \beta$ values in tumors were not yet available. The aim of these breast cancer studies was a more pragmatic one, mainly to decrease the number of visits to the radiotherapy department and to shorten overall treatment time, thereby making adjuvant radiotherapy more convenient for patients. These studies were conducted in countries with centralized radiotherapy structures and long travel distances. It was assumed that a relevant subgroup of patients who were candidates for breast-conserving treatment did in fact choose mastectomy as primary treatment due to the inability to withstand a 6-8-week course of radiotherapy. Therefore, studies were started in the 1990s evaluating an overall shortened treatment time to increase acceptance of adjuvant radiotherapy and reduce costs.

Surprisingly, the latest data from these studies assume an $\alpha / \beta$ value for breast cancer of 3-3.5 Gy which is similar to that of the surrounding normal healthy tissue $[9,10]$. This would mean that fraction size can be changed within a relatively broad range without negative impact on tumor control and late radiation sequelae. Although not expected at the beginning of these trials, the data form a stable theoretical basis for the use of moderate hypofractionation in breast cancer.

\section{Which Studies Support the Effectiveness and Safety of Hypofractionation in Breast Cancer?}

So far, 4 randomized trials have examined a hypofractionated schedule as compared to the standard 5-week fractionation schedule for adjuvant WBI.

The UK START pilot trial assessed dose schedules using either 3 Gy/fraction up to a TD of $39 \mathrm{~Gy}$, or $3.3 \mathrm{~Gy} /$ fraction with a TD of $42.9 \mathrm{~Gy}$ (13 fractions) compared to the standard regimen of $2 \mathrm{~Gy} /$ fraction and a TD of 50 Gy $[11,12]$. The following START-A randomized 2,236 patients to 1 of 3 treatment arms: 3 Gy/fraction (every second day) to a TD of $39 \mathrm{~Gy}, 3.2 \mathrm{~Gy}$ to a TD of $41.6 \mathrm{~Gy}$, and standard normofractionation [13]. START-B compared the standard schedule with a daily hypofractionated schedule using $40 \mathrm{~Gy}$ with a fraction size of 2.66 Gy [14]. It should be noted that in both
START trials $61 \%$ of all patients finally received a sequential boost with 10 Gy (2 Gy/fraction). The boost was always administered after hypofractionated breast irradiation.

After 10-years, all treatment concepts showed equivalent outcomes for hypofractionated radiotherapy compared to the traditional schedule, favoring the use of $40 \mathrm{~Gy}$ in 15 fractions with a low local recurrence rate of 5.6\% [9]. All 3 trials included women with operable T1-3 N0-1 M0 invasive breast cancer and mostly small or medium breast size.

Another trial reproduced these results independently: The Ontario Clinical Oncology Group randomly assigned 1,234 patients to standard WBI or to 42.5 Gy in 16 fractions within 22 days [15]. 1,234 women with invasive breast cancer $(<5 \mathrm{~cm}$, no T3-4, negative margins, node-negative) and without multicentric disease, large breast size, and bilateral breast cancer were included. After 10 years, the risk of recurrence was $6.7 \%$ in the standard group and $6.2 \%$ in the hypofractionated group. Cosmetic outcome was reported as good or excellent with $71.3 \%$ in the standard group and $69.8 \%$ in the hypofractionated group. These studies built the clinical basis for an $\alpha / \beta$ value of breast cancer in the range of 3-3.5 Gy, similar to that of the surrounding healthy tissues (table 1).

\section{Ongoing Controversies Concerning the Hypofractionated Treatment of Breast Cancer}

\section{High-Risk Features}

The majority of patients in the above mentioned trials had estrogen receptor-positive and HER2-negative tumors. It is well known that patients with high-grade tumors have a higher risk of local recurrence after radiation. It is therefore questionable whether hypofractionated WBI is suitable for all patients [1]. The 10 -year update of the START trials including 1,272 women with grade 3 breast cancer showed no difference between hypo- and normofractionation in terms of local recurrence in this group [9], whereas the Ontario trial documented a significantly higher local failure rate in the hypofractionation arm [15], which was somehow considered a statistical fluke. In contrast to the Ontario trial, a sequential boost to the primary tumor site was allowed in the START trials, which may also be a possible explanation for the different treatment results in the high-risk group. Recently, the Ontario group published the results of their analysis of tumor factors predictive for response to hypofractionated versus normofractionated radiotherapy. They stated that tumor grade, histological subtype, and hypoxia did not predict for hypofractionation response, proofing that hypofractionation can be safely administered in all breast cancer patients [16].

\section{Young Age}

The 2011 ASTRO consensus did not recommend hypofractionation in women $<50$ years of age [17] as younger women have a higher risk of local recurrence compared with older patients, and only few younger patients were randomized in those studies at this 
Table 1. Study data on hypofractionation in breast cancer treatment [25]

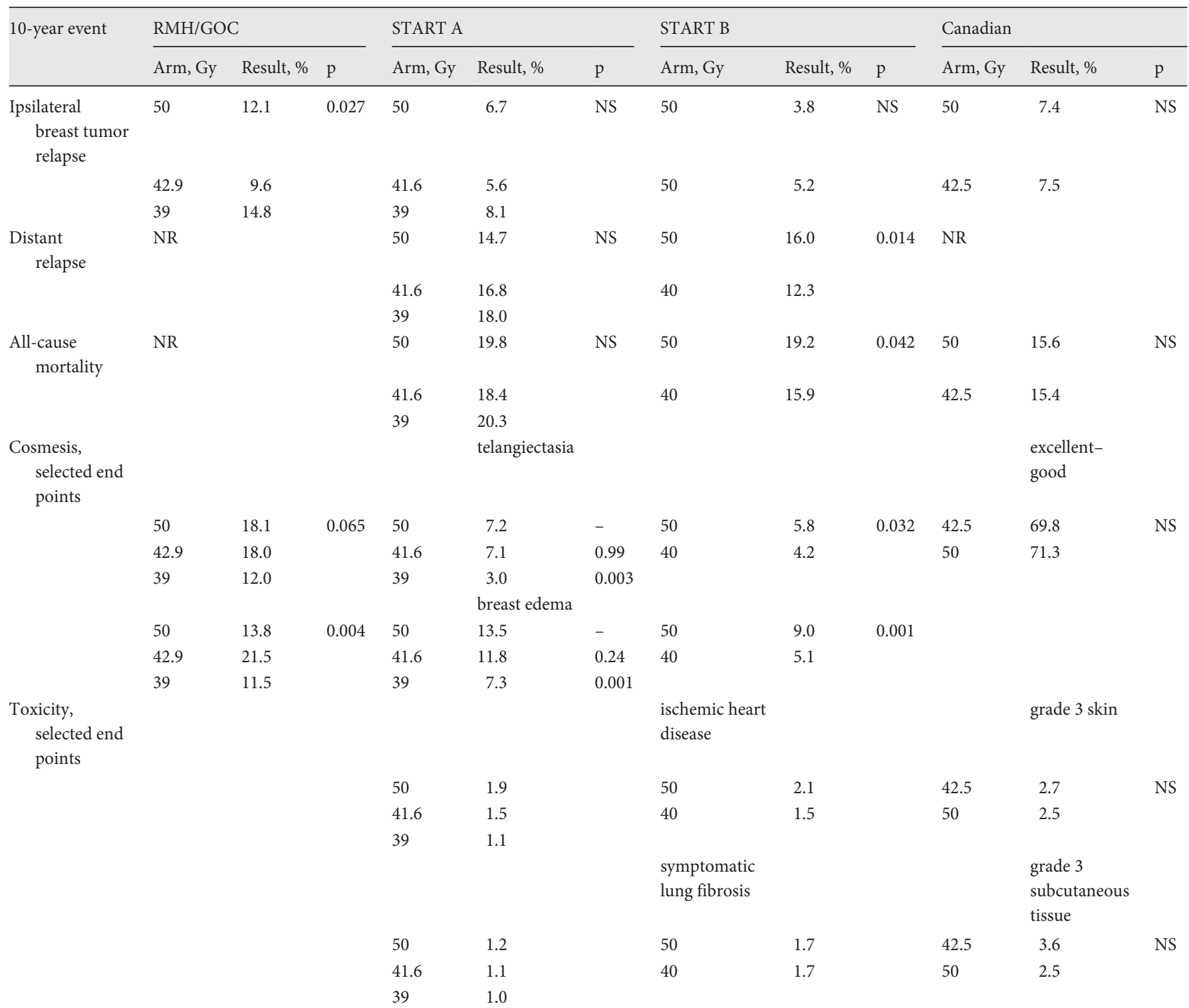

NR = Not reported; NS = not significant; RMH/GOC = Royal Marsden Hospital/Gloucester Oncology Center; START A/B = Standardization of Breast Radiotherapy Trial A/B.

time. However, of the 4 major trials, only the Ontario study stratified by age and detected no significant differences in ipsilateral tumor recurrence, although no boost was applied [15]. The 10-year update of the START trials included 1,398 women $<50$ years of age and demonstrated the effectiveness of hypofractionation in younger patients, even showing a trend towards an improved effect of hypofractionation in this cohort [9].

\section{Regional Nodal Irradiation}

There is only limited experience with hypofractionated regional irradiation due to the small number of women with regional radiotherapy recruited in the reported studies. The safety of the investigated fractionation schemes in view of brachial plexopathy is still under investigation. In the START trials, the only reported plexopathy occurred with the 41.6 Gy fractionation schedule [9].

\section{Cardiotoxicity}

Late cardiac side effects of hypofractionated radiotherapy have only been evaluated to a maximum follow-up of 10 years. Since most radiotherapy-associated cardiac side effects occur after 15 years or more, currently available results must be assessed with caution, especially in women with a long life expectancy and an unhealthy lifestyle. However, no significant cardiotoxicity has been observed in modern normofractionated radiotherapy for breast cancer with current radiotherapy techniques making it now safer than in the past [18]. Recently, Chan et al. [19] reported a 15-year 
follow-up of cardiac injury, comparing hypofractionation versus normofractionation in left-sided early-stage breast cancer patients. In 5,334 patients receiving breast or chest wall irradiation, no statistically significant difference in cardiac mortality was detected.

\section{Implementation of a Simultaneous Integrated Boost in Hypofractionated Radiotherapy of Breast Cancer}

A boost to the primary tumor site improves local control in all age groups. In R0-resected patients, the local recurrence rate is reduced by $4-6.2 \%$ within 10 years [20]. To maintain this important treatment effect in hypofractionated WBI without altering the overall shortened treatment time, 4 studies have been conducted so far in which a simultaneous integrated boost (SIB) was introduced. Scorsetti et al. [21] enrolled 50 women to be treated with an SIBVMAT (volumetric-modulated arc therapy) approach, applying doses of 40.5 Gy to the whole breast and 48 Gy to the primary tumor bed in 15 fractions. They observed 1 grade 3 and 32 grade 1 skin toxicities, with the latter resolving within 3 weeks of treatment completion. No significant changes in cosmetic outcome were observed 3 and 6 months after radiotherapy. Another protocol delivering 40.5 Gy to the breast and 45 Gy as an SIB in 15 fractions over 19 days was investigated by Chada et al. [22]. The 5-year diseasefree survival was $97 \%$, based on a median follow-up of 3.5 years. Acute and late toxicity were low without any grade 3 or 4 skin toxicity after 2 years. A randomized trial by van Parijs et al. [23] compared conventional radiotherapy to hypofractionated tomotherapy with $2.8 \mathrm{~Gy} /$ fraction to the whole breast and an SIB of $0.6 \mathrm{~Gy} /$ fraction in 15 fractions. The primary purpose of this study was to evaluate whether tomotherapy reduces heart and lung toxicity in this setting. They demonstrated a slight trend in favor of tomotherapy, stating that short-course treatment with SIB is feasible with no severe toxicity. The largest prospective investigation so far, a German multicenter phase II study, treated 141 patients with 40 Gy to the whole breast and an SIB of 48 Gy in 16 fractions. The regimen was well tolerated with no grade 3 or 4 adverse events [24]. According to this trial, hypofractionation with SIB seems to be safe and feasible with unchanged quality of life, even though long-term follow- up is still missing. The new prospective multicenter 2 -arm phase III German HYPOSIB trial will provide data on hypofractionated irradiation with SIB with an overall very much shortened treatment time compared to standard radiotherapy. This non-inferiority trial is designed to recruit 1,162 patients in each arm. The study will randomize all patients with histologically confirmed unilateral invasive breast cancer after breast-conserving surgery with an indication for a boost.

\section{Conclusion}

Current clinical results of hypofractionated WBI support its introduction into clinical routine in the near future. Hypofractionation offers an alternative to standard fractionation regimens at least in a subset of patients. There is now a clear radiobiological basis for the use of moderate hypofractionation with a decrease in the number of fractions by about $50 \%$; extreme hypofractionation (e.g. less than 10 fractions) or extremely reduced treatment times (e.g. less than 3 weeks) should, however, be restricted to clinical trials. Moreover, there is limited data on potentially different effects of hypofractionation in subgroups, and it is unclear whether the effects of standard fractionation and hypofractionation are really identical in all tumor subtypes. Delivery of a tumor bed boost in the form of an SIB seems to be safe, feasible, and effective while further reducing the overall treatment time without compromising established excellent overall results. The new HYPOSIB trial will clarify the question of simultaneous boost application and hypofractionation in breast cancer on the basis of a high number of patients. All German radiotherapy facilities are encouraged to participate in the HYPOSIB trial as the study subject is a highly relevant key issue in the development of modern radiotherapy of breast cancer in Germany.

\section{Disclosure Statement}

All authors declare no conflict of interests.

\section{References}

1 Sedlmayer F, Sautter-Bihl ML, Budach W, et al.; Breas Cancer Expert Panel of the German Society of Radiation Oncology (DEGRO): DEGRO practical guidelines: radiotherapy of breast cancer I: radiotherapy following breast conserving therapy for invasive breast cancer. Strahlenther Onkol 2013;189:825-833.

2 Interdisziplinäre S3-Leitlinie für die Diagnostik, Therapie und Nachsorge des Mammakarzinoms, Langversion 3.0, Aktualisierung 2012, AWMF-Register-Nummer: 032-045OL.

3 NCCN (National Comprehensive Cancer Network ${ }^{\circledR}$ ), NCCN Guidelines Version 2.2013 Invasive Breast cancer NCCN (National Comprehensive Cancer Network $\left.^{(}\right)$, Practice Guidelines in Oncology (NCCN Guidelines ${ }^{\circledR}$ ), Breast Cancer Version 3.2013.
4 Scottish Intercollegiate Guidelines Network (SIGN): Treatment of primary breast cancer. Edinburgh: SIGN; 2013. (SIGN publication no. 134). September 2013. www.sign.ac.uk Accessed on 23/04/2014.

5 Darby S, McGale P, Correa C et al.; Early Breast Cancer Trialists' Collaborative Group (EBCTCG): Effect of radiotherapy after breast-conserving surgery on 10year recurrence and 15-year breast cancer death: metaanalysis of individual patient data for 10,801 women in 17 randomised trials. Lancet 2011;378:1707-1716.

6 Thames HD, Bentzen SM, Turesson I, et al.: Time-dose factors in radiotherapy: a review of the human data. Radiother Oncol 1990;19:219-235.
7 Thames HD Jr, Withers HR, Peters LJ, Fletcher GH: Changes in early and late radiation responses with altered dose fractionation: implications for dose-survival relationships. Int J Radiat Oncol Biol Phys 1982;8: 219-226.

8 Koontz BF, Bossi A, Cozzarini C, et al.: A systematic review of hypofractionation for primary management of prostate cancer. Eur Urol 2014;Epub ahead of print.

9 Haviland JS, Owen JR, Dewar JA, et al.; START Trialists' Group: The UK Standardisation of Breast Radiotherapy (START) trials of radiotherapy hypofractionation for treatment of early breast cancer: 10-year follow-up results of two randomised controlled trials. Lancet Oncol 2013;14:1086-1094. 
10 Hennequin C, Dubray B: (Alpha/beta ratio revisited in the era of hypofractionation). Cancer Radiother 2013; 17:344-348

11 Yarnold J, Ashton A, Bliss J, et al.: Fractionation sensitivity and dose response of late adverse effects in the breast after radiotherapy for early breast cancer: longterm results of a randomised trial. Radiother Oncol 2005;75:9-17.

12 Owen JR, Ashton A, Bliss JM, et al.: Effect of radiotherapy fraction size on tumour control in patients with early-stage breast cancer after local tumour excision: long-term results of a randomised trial. Lancet Oncol 2006;7:467-471.

13 Bentzen SM, Agrawal RK, Aird EG, et al.; START Trialists' Group: The UK Standardisation of Breast Radiotherapy (START) Trial A of radiotherapy hypofractionation for treatment of early breast cancer: a randomised trial. Lancet Oncol 2008;9:331-341.

14 Bentzen SM, Agrawal RK, Aird EG, et al.; START Trialists' Group: The UK Standardisation of Breast Radiotherapy (START) Trial B of radiotherapy hypofractionation for treatment of early breast cancer: randomised trial. Lancet 2008;371:1098-1107.

15 Whelan T, Pignol J-P, Levine M, et al.: Long-term results of hypofractionated radiation therapy for breast cancer. N Engl J Med 2010;362:513-520.
16 Bane AL, Whelan TJ, Pond GR, et al.: Tumor factors predictive of response to hypofractionated radiotherapy in a randomized trial following breast conserving therapy. Ann Oncol 2014;25:992-998.

17 Smith BD, Bentzen SM, Correa CR, et al.: Fractionation for whole breast irradiation: an American Society for Radiation Oncology (ASTRO) evidence-based guideline. Int J Radiat Oncol Biol Phys 2011;81:59-68.

18 Nitsche M, Pahl R, Huber K, et al.: Cardiac toxicity after radiotherapy for breast cancer: myths and facts. Breast Care, accepted for publication.

19 Chan EK, Woods R, Virani S, et al.: Long-term mortality from cardiac causes after adjuvant hypofractionated vs. conventional radiotherapy for localized left-sided breast cancer. Radiother Oncol 2015;114:73-78.

20 Poortmans PM, Collette L, Bartelink H, et al.: The addition of a boost dose on the primary tumour bed after lumpectomy in breast conserving treatment for breast cancer. A summary of the results of EORTC 2288110882 'boost versus no boost' trial. Cancer Radiother 2008; $12: 565-570$

21 Scorsetti M, Alongi F, Fogliata A, et al.: Phase I-II study of hypofractionated simultaneous integrated boost using volumetric modulated arc therapy for adjuvant radiation therapy in breast cancer patients: a report of feasibility and early toxicity results in the first 50 treatments. Radiat Oncol 2012;7:145.
22 Chadha M, Woode R, Sillanpaa J, et al.: Early-stage breast cancer treated with 3-week accelerated wholebreast radiation therapy and concomitant boost. Int J Radiat Oncol Biol Phys 2013;86:40-44.

23 Van Parijs H, Miedema G, Vinh-Hung V, Verbanck S, Adriaenssens N, Kerkhove D, Reynders T, Schuermans D, Leysen K, Hanon S, Van Camp G, Vincken W, Storme G, Verellen D, De Ridder M: Short course radiotherapy with simultaneous integrated boost for stage I-II breast cancer, early toxicities of a randomized clinical trial. Radiat Oncol 2012;7:80.

24 Dellas K, Vonthein R, Zimmer J, et al.; ARO Study Group: Hypofractionation with simultaneous integrated boost for early breast cancer: results of the German multicenter phase II trial (ARO-2010-01). Strahlenther Onkol 2014;190:646-653.

25 Fisher CM, Rabinovitch R: Frontiers in radiotherapy for early-stage invasive breast cancer. J Clin Oncol 2014;32:2894-2901. 